Cutts, Q , Maguire, J., Fincher, S. and Parkinson, J. (2021) Forming Community in Computing Science Education with Research in Practice Project Activities. In: UKICER '21: United Kingdom \& Ireland Computing Education Research Conference, Glasgow, UK, 02-03 Sep 2021, p. 12. ISBN 9781450385688.

There may be differences between this version and the published version. You are advised to consult the publisher's version if you wish to cite from it.

(C) The Authors 2021. This is the author's version of the work. It is posted here for your personal use. Not for redistribution. The definitive Version of Record was published in UKICER '21: United Kingdom \& Ireland Computing Education Research Conference, Glasgow, UK, 02-03 Sep 2021, p. 12. ISBN 9781450385688. http://dx.doi.org/10.1145/3481282.3481285.

http://eprints.gla.ac.uk/251970/

Deposited on: 15 September 2021

Enlighten - Research publications by members of the University of Glasgow http://eprints.gla.ac.uk 


\section{Forming Community in Computing Science Education with Research in Practice Project Activities}

\author{
Quintin Cutts \\ School of Computing Science \\ University of Glasgow \\ Glasgow, United Kingdom \\ quintin.cutts@glasgow.ac.uk \\ Sally Fincher \\ School of Computing \\ University of Kent \\ Kent, United Kingdom \\ s.a.fincher@kent.ac.uk
}

\author{
Joseph Maguire \\ School of Computing Science \\ University of Glasgow \\ Glasgow, United Kingdom \\ joseph.maguire@glasgow.ac.uk \\ Jack Parkinson \\ School of Computing Science \\ University of Glasgow \\ Glasgow, United Kingdom \\ jack.parkinson@glasgow.ac.uk
}

\begin{abstract}
The United Kingdom and Ireland Computing Education Research (UKICER) conference is emerging as a leading venue to disseminate research contributions to the community. However, it is important the venue continues to act as an entry point for individuals to participant in computing science education research. Consequently, the present proposal is to offer a new form of collaborative, community-forming activity at the UKICER conference called Research in Practice Project Activities (RIPPAs). The first RIPPA is focused on Spatial Skills and Computing Science and will be offered at UKICER 2021.
\end{abstract}

\section{CCS CONCEPTS}

- Social and professional topics $\rightarrow$ Computing education.

\section{KEYWORDS}

computing science education, review process, community building

ACM Reference Format:

Quintin Cutts, Joseph Maguire, Sally Fincher, and Jack Parkinson. 2021. Forming Community in Computing Science Education with Research in Practice Project Activities. In United Kingdom and Ireland Computing Education Research conference. (UKICER '21), September 2-3, 2021, Glasgow, United Kingdom. ACM, New York, NY, USA, 3 pages. https://doi.org/10.1145/ 3481282.3481285

\section{INTRODUCTION}

The theme for the United Kingdom and Ireland Computing Education Research conference (UKICER) for 2021 is "learning together" and the theme was chosen to emphasise that (1) computing science education researchers can learn from one another and (2) from researchers of education in other disciplines such as mathematics, physics and art. A central aim of the conference is to be both a venue for experts to disseminate rigorous contributions and provide

UKICER '21, September 2-3, 2021, Glasgow, United Kingdom

(C) 2021 Copyright held by the owner/author(s).

This is the author's version of the work. It is posted here for your personal use. Not for redistribution. The definitive Version of Record was published in United Kingdom and Ireland Computing Education Research conference. (UKICER '21), September 2-3, 2021, Glasgow, United Kingdom, https://doi.org/10.1145/3481282.3481285. an entry point for novices to engage with the computing science education research (CER) community.

The collaborative and interdisciplinary nature of CER is driven by necessity. Fincher and Petre argue effective CER researchers often utilise methodologies that are dependent on the research question or problem at hand [5]. An approach that necessitates that such researchers need to form strong cross-institutional communities of sharing practice, experience and knowledge. A strong community that can debate and scrutinise approaches, methodologies as well as exchange knowledge as all individuals are engaged in the same endeavour.

Consequently, vehicles that drive community are arguably particularly important in computing science education research. Fincher and Tenenberg describe the Bootstrapping community-forming project in CER with the aim of (1) establishing a small community of practice that (2) acts an entry point for interested novices that (3) forms a larger community that can establish standards, protocols and practices over a two year period [6]. The project was successful and drove many such other initiatives around the world as well as other projects and venues.

Fincher et al. characterised and identified relationships between some of these different models and activities [4]. Beyond Bootstrapping, another notable model is that of Working Groups (WGs) associated with the annual Innovation and Technology in Computer Science Education (ITiCSE) conference. WGs are proposed by three individuals that act as leaders, shepherding up to 10 recruited computing educators in an intense collaboration to deliver a report that is of interest to the community. The WGs provide an opportunity for individuals from multiple institutions and, most often, several different countries, to rapidly connect, intensively collaborate and produce a valuable contribution. The outcome is a report that typically surveys and analyses a problem of interest to computer educators, rather than empirical research [4].

The potential and capacity for these projects and activities, that drive communities of practice can not be underestimated in terms of impact. Guzdial and du Boulay argue that most CER researchers today in the United States were either participants in such projects or mentored by an individual who participated [7]. Consequently, the 
tailoring and availability of such activities in the United Kingdom and Ireland context could equally have such impact.

A new model adaptation or activity would be to blend the Bootstrapping approach with that of WGs. Blending the Bootstrapping approach of a long-term structure to form expert communities with the WG approach of allowing individuals acting as leaders to propose a specific activity and recruit interested participants to it.

Consequently, from the perspective of building and strengthening the skills base of CER in the United Kingdom and Ireland the contributions of this editorial are to describe:

- the aims and design for Research in Practice Project Activities, a new channel of activity at UKICER,

- the outline of an initial RIPPA focused on research in spatial skills and computing science.

\section{PROPOSAL: RESEARCH IN PRACTICE PROJECT ACTIVITIES}

Research in Practice Project Activities (RIPPAs) are a new form of collaborative, community-forming activity for Computing Science education research and practice with the aim to bring computing educators together to engage in joint work at the United Kingdom and Ireland Computing Education Research (UKICER) conference.

RIPPAs are planned to span several months and participants are expected to commit to participating in a small number of workshops as well as conducting work in their home context, such as collecting data, conducting research over a period of time, or incorporating research into practice.

\subsection{Participation and Commitment}

The specific participation and commitment requirements depend on the RIPPA, but broadly RIPPAs require participants to:

Join an information hour. Prospective participants should join one of several "information hours" to find out about the specific RIPPA and what it involves. This provides an early opportunity to understand the nature of the specific RIPPA, to ask questions and to meet with others interested in the particular activity.

Attend start-up workshop. The initial workshop will introduce the specific RIPPA, the background literature, methodologies and approaches to be used as well as provide an extended opportunity for participants to get to know each other and become familiar with what is happening in each other's contexts, across the community.

Undertake activity. Participants in a RIPPA are expected to undertake some activity as part of the project. This activity could be to conduct research in their own context, or incorporate research into their own practice. Participants share the results of this activity with the group at meetings spaced throughout the project.

Attend interim workshop. The workshop will give participants an opportunity to discuss their experiences and insights from undertaking the activity and start to analyse results in aggregate.

Capstone workshop. The final workshop to pull together any remaining work involved in the activity and form into an artefact (normally one or more papers) that can be disseminated through appropriate channels, such as submission to the United Kingdom and Ireland Computing Education Research (UKICER) conference.

Relax and unwind at a wrap-up dinner. Community is not only built through shared work. The wrap-up meal gives a relaxed opportunity for reflection and exploration of areas of mutual interest with other rippers.

\subsection{Outcomes}

The focus of RIPPAs is the participants and the expectation is that rippers will achieve the following from participating in a RIPPA:

Strengthened knowledge and skills in research and practice. Dependent on the specific RIPPA, the expectation is that participants will strengthen their knowledge and skills. This could be awareness of emerging teaching practice and how to investigate it, appropriate methodologies for CER and/or deepened appreciation of the state of the art in CER.

Strengthened and expanded network of collaborators. Form connections with academics, scholars and researchers from across the computing education community with similar interests and challenges. Having such a network of collaborators is valuable not only for conducting future research investigations but also to gain insight into practice in other institutions as well as creating bonds with other academics to discuss on-going sector challenges and how to solve them.

Publication. The expectation is that outcomes of RIPPAs will be high-quality, multi-institutional studies that draw on authentic data from many different contexts and perspectives. Participants will be authors on these high-quality contributions that are difficult to devise and coordinate for most sole academics.

\section{FIRST ITERATION: SPATIAL SKILLS AND COMPUTING SCIENCE}

The first RIPPA is focused on spatial skills and computing science. Research into spatial skills and computing science has flourished in recent years, with several recent studies exploring the relationship $[1,9,11]$. Spatial skills have been associated with success in computing courses and specific domains of computing (for example, source code navigation [8] and expression evaluation [13]) and training spatial skills has been demonstrated to improve CS outcomes for early-stage students $[2,3,12]$. Low spatial ability also appears to be related to demographic groups with historically lower participation and outcomes in CS programmes: those from lower socio-economic status backgrounds and women [10, 14]. Hence spatial skills research can potentially be of high value for our discipline.

To date these studies have involved only a handful of individual institutions. The goal of this project is to understand more about the role of spatial skills across a one-year period for entry-level CS students across multiple institutions. In particular, the RIPPA would like to determine whether correlations between spatial ability and success in CS hold across institutions, whether spatial ability changes over a period of CS instruction and how spatial ability exhibits in different demographic groups. 


\subsection{Overview of Activity}

Participation in this RIPPA requires participants to:

- Conduct three spatial skills tests (each lasting about half an hour) across the academic year with students in introductory computing

- Collect naturally occurring student data, including final course grades, course choices and demographic information

- Apply for ethical approval (if required) but the leading RIPPA institution in this instance is seeking blanket approval.

- Contribute to analysis and dissemination activities

The input from students required for this RIPPA is limited: testing their spatial skills and collecting grades and demographics. Thus, the work for each participant is low, but the potential contribution of the collective work is high.

\section{CONCLUSION}

The interdisciplinary and collaborative nature of CER ensures that vehicles that engage participants, expand participation and elevate practice are crucial to ensuring an effective CER community. In the United Kingdom and Ireland context, RIPPAs are proposed to engage computing educators from across the region to conduct research in the form of collecting data, contributing to analysis and disseminating it to others. RIPPA is a start, rather than an end. The expectation is that through engagement with computing educators, the base of experienced and effective researchers in CER will grow, leading to more RIPPAs and other such activities in future.

\section{REFERENCES}

[1] Ryan Bockmon, Stephen Cooper, Jonathan Gratch, Jian Zhang, and Mohsen Dorodchi. 2020. Can Students' Spatial Skills Predict Their Programming Abilities? In Proceedings of the 2020 ACM Conference on Innovation and Technology in Computer Science Education. ACM, Trondheim Norway, 446-451. https://doi. org $/ 10.1145 / 3341525.3387380$

[2] Ryan Bockmon, Stephen Cooper, William Koperski, Jonathan Gratch, Sheryl Sorby, and Mohsen Dorodchi. 2020. A CS1 Spatial Skills Intervention and the Impact on Introductory Programming Abilities. In Proceedings of the 51st ACM
Technical Symposium on Computer Science Education. ACM, Portland OR USA, 766-772. https://doi.org/10.1145/3328778.3366829

[3] Stephen Cooper, Karen Wang, Maya Israni, and Sheryl Sorby. 2015. Spatial Skills Training in Introductory Computing. In Proceedings of the eleventh annual International Conference on International Computing Education Research. ACM, Omaha Nebraska USA, 13-20. https://doi.org/10.1145/2787622.2787728

[4] Sally Fincher, Raymond Lister, Tony Clear, Anthony Robins, Josh Tenenberg, and Marian Petre. 2005. Multi-institutional, multi-national studies in CSEd research: Some design considerations and trade-offs. In Proceedings of the first international workshop on computing education research. 111-121.

[5] Sally Fincher and Marian Petre. 2004. Computer science education research. CRC Press.

[6] Sally Fincher and Josh Tenenberg. 2006. Using theory to inform capacity-building: Bootstrapping communities of practice in computer science education research. Fournal of Engineering Education 95, 4 (2006), 265-277.

[7] Mark Guzdial and Benedict du Boulay. 2019. The History of Computing. The Cambridge handbook of computing education research (2019), 11.

[8] Sue Jones and Gary Burnett. 2007. Spatial skills and navigation of source code. In Proceedings of the 12th annual SIGCSE conference on Innovation and technology in computer science education - ITiCSE '07. ACM Press, Dundee, Scotland, 231. https://doi.org/10.1145/1268784.1268852

[9] Lauren E. Margulieux. 2019. Spatial Encoding Strategy Theory: The Relationship between Spatial Skill and STEM Achievement. In Proceedings of the 2019 ACM Conference on International Computing Education Research. ACM, Toronto ON Canada, 81-90. https://doi.org/10.1145/3291279.3339414

[10] Miranda C. Parker, Amber Solomon, Brianna Pritchett, David A. Illingworth, Lauren E. Marguilieux, and Mark Guzdial. 2018. Socioeconomic Status and Computer Science Achievement: Spatial Ability as a Mediating Variable in a Novel Model of Understanding. In Proceedings of the 2018 ACM Conference on International Computing Education Research. ACM, Espoo Finland, 97-105. https: //doi.org/10.1145/3230977.3230987

[11] Jack Parkinson and Quintin Cutts. 2018. Investigating the Relationship Between Spatial Skills and Computer Science. In Proceedings of the 2018 ACM Conference on International Computing Education Research. ACM, Espoo Finland, 106-114. https://doi.org/10.1145/3230977.3230990

[12] Jack Parkinson and Quintin Cutts. 2020. The Effect of a Spatial Skills Training Course in Introductory Computing. In Proceedings of the 2020 ACM Conference on Innovation and Technology in Computer Science Education. ACM, Trondheim Norway, 439-445. https://doi.org/10.1145/3341525.3387413

[13] Jack Parkinson, Quintin Cutts, and Steve Draper. 2020. Relating Spatial Skills and Expression Evaluation. In United Kingdom \& Ireland Computing Education Research conference. ACM, Glasgow United Kingdom, 17-23. https://doi.org/10. $1145 / 3416465.3416473$

[14] David H. Uttal, Nathaniel G. Meadow, Elizabeth Tipton, Linda L. Hand, Alison R. Alden, Christopher Warren, and Nora S. Newcombe. 2013. The malleability of spatial skills: A meta-analysis of training studies. Psychological Bulletin 139, 2 (March 2013), 352-402. https://doi.org/10.1037/a0028446 